\title{
CONSIDERACIONES SEMANTICAS SOBRE LÓGICA DEÓNTICA, CON ESPECIAL REFERENCIA A LA JURISPRUDENCIA
}

ULISES SCHMILL Universidad Nacional Autónoma de Mérico

En este trabajo se presenta una semántica para ciertas lógicas deónticas. La base del mismo lo constituye el método de los conjuntos modelo, desarrollado por Hintikka, tal como ha sido presentado para la lógica modal en el libro de P. D. Snyder [7]. Asimismo, se propondrá, con gran analogía al trabajo de A. R. Anderson [1], una semántica de ciertas fórmulas con operadores deónticos reiterados.

1.1. Partimos de un lenguaje formalizado al que denominaremos L. Este lenguaje sólo comprende el cálculo proposicional. En este trabajo se utilizará la notación polaca de Lukasiewicz, con una ligera modificación.

Como vocabulario de $\mathbf{L}$ se tiene:

i) constantes proposicionales: $p, q, \mathbf{r} \ldots \mathbf{p}^{\prime}, \mathrm{q}^{\prime}, \mathbf{r}^{\prime} \ldots$

ii) constantes lógicas, simbolizadas por letras capitales latinas: A (alteración: ó), $\mathrm{K}$ (conjunción: y), C (condicional: si ... entonces) y- (negación: no).

Por fórmula atómica entendemos la fórmula bien formada (wff) más simple: en nuestro caso son las constantes proposicionales $\mathrm{p}, \mathrm{q}, \mathrm{r}, \mathrm{s}, \ldots$ etc.

Por fórmula básica entendemos una fórmula atómica o la negación de una fórmula atómica: $p, q, \bar{p}, \bar{q}, \ldots$

Las reglas de formación de las fórmulas bien formadas (wff) son las usuales con la notación polaca. La única va. riante es la de que el signo de negación siempre lo colocare- 
mos sobre el signo que se encuentra en el extremo izquierdo de la fórmula negada.

1.2. Conjuntos modelos. Muchas ramas de la lógica son estudiadas con dos tipos distintos de métodos: los sintácticos y los semánticos. Los métodos semánticos han sido especialmente fértiles en sus resultados' cuando se han aplicado a diversos cálculos. En la lógica modal, los métodos semánticos han resultado, además, sumamente útiles para la acla. ración de diversos conceptos.

Se distinguirá en este ensayo entre el lenguaje objeto y el metalenguaje correspondiente. Cómo se usarán los conjuntos modelo de Hintikka y los sistemas modelo, tal como se definirán más adelante, utilizaremos letras griegas ' $\alpha$ ', ' $\beta$ ' . . . para representar las.wff del lenguaje-objeto.

En el metalenguaje se hará uso de $\lambda, \mu, \nu, \kappa, \kappa_{1}, \ldots$ para nombrar a los conjuntos modelo. 0 será el nombre de un conjunto'modelo en particular que se supone fijado de antemano. $(\alpha)$ representa a la wff $\alpha$ como contenida en un con. junto modelo; $\varepsilon$ es usado como el símbolo de pertenencia a un conjunto modelo; $\$$ como el símbolo de no pertenencia a un conjunto modelo de una wff.

Este vocabulario metalingüístico facilitará la descripción de las relaciones entre un cálculo o sistema formal y su mo. delo o interpretación semántica. Se escribirá:

$$
\text { (a) } \varepsilon \mu
$$

para significar que la fórmula correspondiente a $\alpha$ pertenece al conjunto modelo $\mu$. Si $\alpha$ pertenece al conjunto modelo fijo, se escribirá $(\alpha) \varepsilon 0$. Si no pertenece $\alpha$ al conjunto modelo fijo 0 , se escribirá $(\alpha) \notin 0$.

Un conjunto modelo $\mu$ es un conjunto de fórmulas que sa. tisfacen las siguientes condiciones (ssi es el símbolo en el metalenguaje correspondiente a 'si y sólo si') :
(C.1)
$(\bar{\alpha}) \varepsilon \mu$ ssi $(\alpha) \notin \mu$
(C.N)
$(\overline{\bar{\alpha}}) \varepsilon \mu$ ssi
$(\bar{\alpha}) \notin \mu$
(C.NN) 
(C.3) $(\mathrm{K} \alpha \beta) \varepsilon \mu$ ssi $(\alpha) \varepsilon \mu$ у $(\beta) \varepsilon \mu$ (C.K)

(C.4) $(\mathrm{A} \alpha \beta) \varepsilon \mu$ ssi $(\alpha) \varepsilon \mu$ ó ( $\beta) \varepsilon \mu$ (C.A)

(C.5) $(\mathrm{C} \alpha \beta) \varepsilon \mu$ ssi $(\bar{\alpha}) \varepsilon \mu$ ó $(\beta) \varepsilon \mu$ (C.C)

(C.6) $(\overline{\mathrm{K}} \alpha \beta) \varepsilon \mu$ ssi $(\bar{\alpha}) \varepsilon \mu$ o $(\bar{\beta}) \varepsilon \mu(\mathrm{C} . \overline{\mathrm{K}})$

(C.7) $(\overline{\mathrm{A}} \alpha \beta) \varepsilon \mu$ ssi $(\bar{\alpha}) \varepsilon \mu$ y $(\bar{\beta}) \varepsilon \mu(\mathrm{C} . \overline{\mathrm{A}})$

(C.8) $(\overline{\mathrm{C}} \alpha \beta) \varepsilon \mu$ ssi $(\alpha) \varepsilon \mu$ у $(\bar{\beta}) \varepsilon \mu($ C. $\overline{\mathrm{C}})$

Un conjunto modelo puede entenderse como una descripción parcial de un posible estado de cosas.

I.3. Sistema modelo. Un sistema modelo s.m. es un triple ordenado $(G, \Omega, H)$, en donde $\Omega$ es un conjunto no vacío (de conjuntos modelo), $G \varepsilon \Omega$ y $G=0$ (el conjunto modelo fijo); $H$ es una relación de dos lugares, definida sobre $\Omega$, llamada relación de alternatividad. $H \lambda \mu$ se utilizará como '(el conjunto modelo) $\lambda$ es H-alternativo al (conjunto modelo) $\mu$ '.

Un conjunto modelo $\mu$ en un sistema modelo s.m. satisface las siguientes condiciones, además de las enumeradas anteriormente:

(C.9) $(\mathrm{M} \alpha) \varepsilon \mu \varepsilon \Omega$ ssi $\Sigma \lambda \mathrm{KH} \lambda \mu(\alpha) \varepsilon \lambda \quad$ (C.M)

$\begin{array}{lll}\text { (C.10) (L } \alpha) \varepsilon \mu \varepsilon \Omega \text { ssi } \Pi \lambda C H \lambda \mu(\alpha) \varepsilon \lambda & \text { (C.L) }\end{array}$

(C.M) puede leerse diciendo: 'es posible $\alpha$ ', es una de las sentencias contenidas en el conjunto modelo $\mu$, que es miembro de $\Omega$ si y sólo si hay un conjunto de sentencias $\lambda$ que constituye un conjunto modelo H-alternativo a $\mu$ y $\alpha$ es miembro del conjunto modelo $\lambda$.

(C.L) puede leerse: 'es necesario $\alpha$ ', es una de las sentencias contenidas en el conjunto modelo $\mu$ que es miembro de $\Omega$ si y sólo si para todo conjunto $\lambda$ si $\lambda$ es $H$-alternativo de $\mu$, entonces $\alpha$ es miembro de $\lambda$.

(C.M) interpreta nuestras intuiciones de que ' $\alpha$ es posible' si $\alpha$ es verdadera en un mundo alternativo del nuestro y ' $\alpha$ es necesario' si $\alpha$ es verdadera en todos los mundos posibles.

$\mathrm{Si}$ la relación $\mathrm{H}$ entre conjuntos modelo es reflexiva, el sistema modelo s.m. constituye una interpretación del sistema 
T de Feys de lógica modal, equivalente al sistema $M$ de von Wright. Cuando satisfaga esta condición se denominará este sistema modelo 'un sistema modelo $\mathrm{M}$ '.

Se obtendrá un 'sistema modelo S4' si se establece la condición de que la relación $H$ entre conjuntos modelo sea reflexiva y transitiva.

Se obtendrá un 'sistema modelo broweriano' si se establece la condición de que la relación $\mathrm{H}$ sea reflexiva y simétrica y si es una relación reflexiva, transitiva y simétrica, se obtendrá el 'sistema modelo S5'.

II

II.1. Posturas no reduccionistas (n.r) y reducionistas ( $r$ ) de lógica deóntica. En general existen, por lo menos, dos posibilidades en relación con una formalización de los conceptos normativos:

(a) la construcción de una lógica deóntica, en el sentido definido por D'. Føllesdal y R. Hilpinen [2] como 'el estudio de las sentencias en las que solamente palabras lógicas y expresiones normativas ocurren esencialmente', distinta (como extensión) de la lógica proposicional (Hintikka) o de la lógica modal alética (A. R. Anderson), por la introducción, en este último caso, de cierta constante proposicional y un. axioma que más adelante se precisarán. (Postura n.r).

(b) la construcción de la lógica deóntica en la que los operadores deónticos se reducen a expresiones de una lógica modal alética (A. R. Anderson). (Postura r).

En el caso (a), o sea, en la postura n.r, se presentan a su vez dos posibilidades:

(i) construir la lógica deóntica por medio de la introducción ex novo de los operadores deónticos $\mathrm{O}$ y $\mathrm{P}$ a la lógica proposicional, definida con las condiciones (C.1) - (C.8). 
(ii) construir la lógica deóntica adicionando a la lógica modal alética, definida con las condiciones (C.1)(C.10), una constante proposicional ' $\mathrm{S}$ ' (que se interpreta como sanción), y, además, un axioma que establece la negación de S como posible: Ms̄.

Veamos cada una de estas posturas.

En el caso (i), conforme a Hintikka, una sentencia 'Pp' supone que

Cuando afirmamos que una permisión se da en un mundo, no hablamos únicamente de este mundo, sino que hablamos en algo parecido al modo subjuntivo o contrafáctico. Hablamos de lo que pudo ser o pudo suceder; decimos que algo pudo haberse realizado o haber sido el caso sin vio. larse ninguna obligación (norma). Esto implica que no podemos formular las condiciones sobre la presencia de Pp en la descripción $\mu$ de un mundo posible en términos exclusivamente de $\mu$. Además de $\mu$, debemos considerar otra descripción de un mundo posible relacionada con $\mu$ de cierta manera. Esta manera será expresada diciendo que $\mu^{*}$ es una alternativa deóntica de $\mu$. (Hintikka [3], p. 70).

Por ello formula su condición sobre el operador deóntico 'P', de la siguiente manera:

(C.P) Si Pp\& $\mu$, entonces por lo menos para una alternativa deóntica $\mu^{*}$ de $\mu$ tenemos $\mathrm{p} \varepsilon \mu^{*}$.

Y en el caso del operador deóntico ' 0 ', la condición es: (C. $\left.0^{*}\right)$ Si $0 \mathrm{p} \varepsilon \mu$ у $\mu^{*}$ es una alternativa deóntica de $\mu$, entonces pe $\mu^{*}$.

Además formula otras dos condiciones:

(C.0 rest) Si $0 \mathrm{p} \varepsilon \mu^{*}$ y si $\mu^{*}$ es una alternativa deóntica a algún conjunto modelo $\mu$, entonces $p \varepsilon \mu^{*}$. 
(C.00*) Si $0 \mathrm{p} \varepsilon \mu$ y si $\mu^{*}$ es una alternativa deóntica a $\mu$, entonces $0 \mathrm{p} \varepsilon \mu^{*}$.

Hintikka dice que a $\mu *$ como alternativa deóntica de $\mu$ debe representársele "como una descripción de la situación en la que asumió que $p$ fue el caso, con el propósito de mostrar que puede ser el caso mientras todas las obligaciones son cumplidas" (Hintikka [3], p. 70).

De lo anterior se desprende que Hintikka piensa que tanto $\mu$ (el mundo (¿normativo?) actual) como sus alternativas deónticas $\mu *$ reúnen las condiciones de los conjuntos mode. lo deónticos. Es decir, $\mu$ y $\mu *$ son construidos con nuestras condiciones (C.1)-(C.8) —"se asume, claro está, que las alternativas deónticas de conjuntos modelo son también conjuntos modelo" (Ibidem) - y además, las condiciones acabadas de enumerar. No es necesaria la inclusión de (C.9) y (C.10), porque en la lógica deóntica de Hintikka no interviene la lógica modal como incluida.

Si representamos a los conjuntos modelo proposicionales con $\Pi_{i}$ y a los deónticos como $\delta_{i}$, se tiene:

$$
\left\{\Pi_{1}\right\}=\Omega \text { prop } \subset\left\{\delta_{i}\right\}=\Omega \text { deo }
$$

(el conjunto de los conjuntos modelo proposicionales constitutivos del sistema modelo proposicional está incluido propiamente en el conjunto de los conjuntos modelo deónticos constitutivo del sistema modelo deóntico).

En el caso (ii), la situación es similar, pues la lógica deóntica correspondiente constituye una extensión de la lógica modal alética, tal como en el caso (i) la lógica deóntica es una extensión de la lógica proposicional.

Se obtiene una lógica deóntica si se adiciona a la lógica modal alética una constante proposicional ' $s$ ' (que se interpreta como sanción), cuya negación es posible y se define Op de la siguiente manera:

E0pLC̄̄s

cuya traducción dice: 
'es obligatorio $p$ ssi no $p$ implica estrictamente la sanción'.

Debe observarse que los operadores ' $\mathrm{P}$ ' y ' 0 ' funcionan de igual manera en la postura n.r, extensión del cálculo proposicional.

La siguiente Tabla lo demuestra:

Tabla I

\section{(A)}

Postura n.r extensión del cálculo proposicional

(a) $0 \mathrm{p}=\overline{\mathrm{P}} \overline{\mathrm{p}}$

(b) $\mathrm{Php}=\overline{\mathrm{P}} \mathbf{\mathrm { p }}$
(B)

Postura n.r extensión de lógica modal alética
(a) $\mathrm{P}_{\mathrm{p}}=\mathrm{MKp} \overline{\mathrm{s}}$
(a) ${ }^{\prime \prime} 0 \mathrm{p}=\overline{\mathrm{P}} \overline{\mathrm{p}}=\mathrm{MK} \overline{\mathrm{ps}}=\mathrm{LC} \overline{\mathrm{ps}}$
(b) ${ }^{\prime} \mathrm{Php}=\overline{\mathrm{P}}_{\mathrm{p}}=\mathrm{LC} \mathrm{ps}$ :

En la posición n.r (A) si ' $\mathrm{P}$ ' se toma como operador básico no definido, 'Op' se define como '⿳亠丷厂 no está permitida' y 'Php' se define como 'p no está permitida', i.e., está prohibida.

En cambio, en la postura no reduccionista (B), 'Pp' se define como 'es posible que ambas $\mathrm{p}$ y no se suscite la sanción', o sea, en caso de que ' $p$ ' sea verdadera o se realice, la sanción no se aplique.

Pero ' $0 p$ ' tiene más significación, aunque su funcionamiento formal sea el mismo: puesto en relación con la san. ción, 'Op' significa que $\overline{\mathrm{p}}$ implica estrictamente la sanción, o sea, que es imposible la no realización de $p$ y no se suscite la sanción y en relación con 'Php', $(b)^{\prime}$ ' señala que $\mathrm{p}$ está prohibida, i.e., no permitida, si $\mathrm{p}$ implica estrictamente la sanción, si p está sancionada, o sea, si es la condición de la sanción.

El funcionamiento formal en ambas posiciones es idéntico. Resulta elemental hacer las mismas consideraciones para el caso de que las posturas n.r se construyan con base en 0 , en lugar de con base en $\mathbf{P}$.

$\mathrm{El}$ caso (b) (postura $\mathbf{r}$ ) tiene una gran analogía con la postura n.r (B), pues su única diferencia consiste en que en 
lugar de adicionar $\mathrm{S}$ a la lógica modal alética y un axioma $\mathrm{Ms}$, se define $\mathrm{S}$ con una fórmula proposicional que debe reunir las dos características siguientes:

1) que no se pueda demostrar que su negación es posible

2) que no sea demostrablemente verdadera la fórmula y su negación.

Si las dos condiciones anteriores se reúnen, el axioma $\mathbf{M} \bar{s}$ de la posición n.r (B) se elimina y se obtiene la reducción de la lógica deóntica modal alética.

A. R. Anderson [1] ha demostrado que la fórmula $\mathrm{KMB} B$ reúne esas dos condiciones mencionadas, pues ella no es un teorema del cálculo modal alético ni tampoco lo es su negación $\bar{K} M \bar{B} B$; pero sí es un teorema $\mathrm{MK} \overline{\mathrm{K}} \overline{\mathrm{BB}}$, por lo que define $\mathrm{S}$ como $\mathrm{KM} \overline{\mathrm{B}} \mathrm{B}$. Por tanto, las definiciones $(a)^{\prime},(a)^{\prime \prime} \mathrm{y}$ $(b)^{\prime}$ son válidas en esta posición $r$.

III

III.1. Semántica deóntica de la postura n.r andersoniana. Resulta interesante la aplicación de las condiciones (C.9) y (C.10) (véase I.3. de este ensayo) a las posturas de A. R. Anderson, pues ello proporcionará una interpretación de di. versos problemas de la jurisprudencia.

III.1.1. En lo que hemos denominado posición n.r (B) (Tabla I), a la lógica modal alética se le adiciona una constante proposicional S y un axioma Ms̄. Con esta base se define:

$$
\mathrm{Pp}_{\mathbf{p}}=\mathrm{MK} \mathbf{\mathrm { s }}
$$

(es posible $\mathrm{p}$ y no $s$, i.e., $\mathrm{p}$ no está sancionada), $\mathrm{MK} \mathrm{p} \overline{\mathrm{s}}$ es un enunciado perteneciente a un conjunto modelo específico, al que denominaremos $\kappa_{0}$, que describe el contenido de una norma específica, diciendo que p está permitida. Por lo tanto, es un enunciado perteneciente al sistema de la Jurisprudencia.

Por tanto, se escribirá:

$$
\mathrm{Pp}=(\mathrm{MKp} \overline{\mathrm{s}}) \kappa_{0}
$$


Aplicando la condición (C.M) se obtiene:

(MP) $(\mathrm{MKp} \overline{\mathrm{s}}) \kappa_{0}$ ssi $\Sigma \kappa_{1} \mathrm{KH} \kappa_{1} \kappa_{0} \mathrm{~K}(\mathrm{p}) \kappa_{1}(\overline{\mathrm{s}}) \kappa_{1}$

Este es un enunciado metalingüístico que traduce el enun. ciado del lenguaje objeto, en nuestro caso, la jurisprudencia positiva, $(M K \overline{p s})$, en fórmulas metalingüísticas que no contienen operadores modales, pero que describen la semántica de la lógica modal que se ha expuesto.

Regla de Traducción: Si Q representa un operador de dos lugares del cálculo proposicional, se tiene esta regla de traducción al metalenguaje de la jurisprudencia:

(RT) $(Q \alpha \beta) \mu$ se traduce $Q(\alpha) \mu(\beta) \mu$

La negación, en la traducción se coloca fuera del paréntesis y afecta al signo más a la izquierda en los pasos de la traducción.

Se mostrarán detalladamente los pasos de la traducción de (MP) :

(1) (MKp̄s)

Aplicando la condición (C.9) se obtiene

(2) $\Sigma \kappa_{1} \mathrm{KH}_{\kappa_{1}} \kappa_{0}(\mathrm{Kp} \overline{\mathbf{s}}) \kappa_{1}$

Este es el momento de aplicar la regla de traducción (RT), pues lo único que está entre paréntesis tiene una forma $Q \alpha \beta$

(3) $\Sigma \kappa_{1} \mathrm{KH} \kappa_{1} \kappa_{0} \mathrm{~K}(\mathrm{p}) \kappa_{1}(\overline{\mathbf{s}}) \kappa_{1}$

(AQJP)

Con lo cual la traducción del metalenguaje queda completa.

La fórmula (3) puede expresarse diciendo que existe un conjunto modelo $\kappa_{1}$, que es $\mathrm{H}$-alternativo al conjunto modelo $\kappa_{0}$ y $(\mathrm{p}) \varepsilon \kappa_{1}, y(\bar{s}) \varepsilon \kappa_{1}$, o sea que el conjunto modelo $\kappa_{1}$ contiene a $\mathrm{Kp} \overline{\mathbf{s}}$.

Proponemos, en consecuencia, la siguiente interpretación: Jurisprudencialmente, consideramos al Derecho formado por un conjunto de normas $\left\{\mathbf{n}_{1}, \mathbf{n}_{2}, \mathbf{n}_{3}, \ldots \mathrm{n}_{\mathbf{n}} \ldots\right\}$. 
Es función de la Jurisprudencia establecer teoréticamente el sistema de tales normas. Tal sistema de normas es el correlato del sistema de enunciados de la jurisprudencia descriptivo de las normas $\left\{\mathbf{n}_{1}, \mathbf{n}_{2}, \mathbf{n}_{3}, \ldots \mathbf{n}_{\mathbf{n}} \ldots\right\}$. La Jurisprudencia es un conjunto de enunciados $\left\{\kappa_{0}, \kappa_{1}, \kappa_{2}, \ldots \kappa_{\mathrm{n}} ..\right\}$ que determinan el contenido de las normas jurídicas. Conforme a esto, $\boldsymbol{\kappa}_{i}$ constituye un conjunto modelo descriptivo de la norma $n_{i}$. Cada $\kappa_{i}$ reúne las condiciones (C.1)-(C.10).

Pero tenemos, además, una traducción metalingüística del lenguaje jurisprudencial.

El metalenguaje jurisprudencial al que denominaremos (AQJ) constituye lo que tradicionalmente se ha denominado Jurisprudencia General o Jurisprudencia Pura.

Con estos elementos, la fórmula (AQJP), expresa intuiti. vamente lo siguiente:

La proposición jurídica $\kappa_{0}$ que determina el contenido de la norma jurídica $\mathbf{n}_{0}$ diciendo que $\mathbf{p}$ está permitida, significa conforme a la semántica introducida, que existe una proposición jurídica $\kappa_{1}$ dependiente de $\kappa_{0}$ que determina que la realización de $p$ no está sancionada.

0 expresando esto desde el punto de vista de la proposición jurídica:

La norma $\mathbf{n}_{0}$ que permite $\mathrm{p}$ determina el contenido de la norma $n_{1}$ dependiente de $n_{0}$ en el sentido de que $p$ no ha de sancionarse, o sea $\mathrm{Kp} \overline{\mathrm{s}}$.

Veamos ahora el principio de prohibición:

$\mathrm{Php}=\overline{\mathbf{P}} \mathbf{p}=\overline{\mathbf{M}} K_{\mathrm{p}} \overline{\mathbf{s}}$ (AQJPh)

Por tanto,

$(\overline{\mathrm{M}} \mathrm{p} \overline{\mathbf{s}}) \kappa_{0}$

$\bar{\Sigma} \kappa_{1} K H \kappa_{1} \kappa_{0}(K p \bar{s}) \kappa_{1}$

$\bar{\Sigma} \kappa_{1} K H \kappa_{1} \kappa_{0} K(p) \kappa_{1}(\bar{s}) \kappa_{1}$ 
Expresando en términos positivos, por transformaciones elementales, se tiene:

$$
\overline{\mathrm{M}} \mathrm{Kp} \overline{\mathrm{s}}=\overline{\mathrm{L}} \overline{\mathrm{K}} \mathrm{p} \overline{\mathrm{s}}=\mathrm{LA} \overline{\mathrm{p} s}=\mathrm{LC} \mathrm{ps}
$$

Por tanto, se tiene:

$$
\begin{aligned}
& (\mathrm{LCps}) \kappa_{0} \\
& \prod \kappa_{1} \mathrm{CH} \kappa_{1} \kappa_{0}(\mathrm{Cps}) \kappa_{1} \\
& \prod \kappa_{1} \mathrm{CH} \kappa_{1} \kappa_{0} \mathrm{C}(\mathrm{p}) \kappa_{1}(\mathrm{~s}) \kappa_{1} \quad \text { (AQJPh) }
\end{aligned}
$$

Esto establece una condición central para todos los conjuntos modelo $\kappa_{1}, \mathrm{H}$-alternativos a $\kappa_{0}$. Para todo conjunto modelo $\kappa_{1} H$-alternativo a $\kappa_{0}$, si tiene contenido (p), i.e. (p) $\varepsilon \kappa_{1}$, entonces, ha de contener también (s), i.e. (s) $\varepsilon \kappa_{1}$. Lo cual significa que toda proposición jurídica $\kappa_{1}$ dependiente de $\kappa_{0}$ ha de considerar p como sancionado.

Desde el punto de vista de la Jurisprudencia positiva, se diría que toda norma $n_{1}$ dependiente de $n_{0}$ no puede tener otro contenido que el de Cps.

Véase ahora el principio de la obligación.

$$
0_{\mathrm{p}}=\overline{\mathrm{P}} \overline{\mathrm{p}}=\overline{\mathrm{M}} \mathrm{K} \overline{\mathrm{ps}}
$$

Lo cual, haciendo la traducción correspondiente, resulta en

$$
\left.\bar{\Sigma}_{\kappa_{1}} \mathrm{KH}_{\kappa_{1} \kappa_{0}} \mathrm{~K}(\overline{\mathbf{p}}) \boldsymbol{\kappa}_{1}(\overline{\mathrm{s}}) \boldsymbol{\kappa}_{1} \quad \text { (AQJO) }\right)^{\prime}
$$

Haciendo las transformaciones corresondientes:

$$
0 \mathrm{p}=\overline{\mathrm{M}} \mathrm{Kp} \overline{\mathrm{s}}=\mathrm{LC} \overline{\mathrm{p}} \mathrm{s}
$$

LCps se traduce en $\Pi \kappa_{1} \mathrm{CH} \kappa_{1} \kappa_{0} \mathrm{C}(\overline{\mathrm{p}}) \kappa_{1}(\mathrm{~S}) \kappa_{1} \quad$ (AQJ0)

Esta fórmula establece también otra condición esencial para los conjuntos modelo $\kappa_{1} \mathrm{H}$-alternativos a $\kappa_{0}$. Si en $\kappa_{0}$ existe el enunciado 0 p, todo conjunto modelo $\kappa_{1} \mathrm{H}$-alternativo a $\kappa_{0}$ si tiene contenido $(\overline{\mathbf{p}})$, entonces debe tener contenido también (S), i.e., $\overline{\mathrm{p}}$ siempre debe estar sancionado.

Todo lo anterior debe interpretarse del siguiente modo:

Se tiene un sistema modelo s.m. como un triple $\left(\kappa_{0}, \mathrm{~K}, \mathrm{H}\right)$ 
donde $\mathrm{K}$ es un conjunto, $\kappa_{0} \varepsilon \mathrm{K}$ y $\mathrm{H}$ es una relación reflexiva. No dejamos que $\mathrm{H}$ sea transitiva, pues con estos elementos no puede interpretarse la reiteración de operadores deónticos de un modo adecuado. Para ello se requiere la creación de un cálculo semejante, que se dará después, con una interpretación intuitiva distinta. Por lo tanto, esta lógica deóntica constituye un sistema deóntico $\mathrm{M}$, al que denominaremos DM.

$\kappa_{0}$ es un conjunto modelo específico, señalado particularmente como aquel que describe el contenido de una norma (jurídica) específica. Los demás conjuntos modelo $\kappa_{1}, \kappa_{2}, \ldots \varepsilon \mathrm{K}$ se entienden como proposiciones jurídicas dependientes de la proposición jurídica $\kappa_{0}$.

Estas condiciones determinan el criterio de consistencia material entre el contenido deóntico de las proposiciones (jurídicas) dependientes de una proposición (juridica) específica.

Es pertinente en este lugar recordar la concepción de H. Kelsen sobre la gradación del orden jurídico, i.e., la concepción de que el orden normativo jurídico (dinámico) consiste en un conjunto de normas relacionadas entre sí por una relación de condicionamiento, relación que se expresa como jerarquía, si se utiliza la metáfora especial de "norma superior" y "norma inferior".

Denominase "norma superior" a aquella que determina tanto el proceso de creación de otra norma (la norma inferior) como su contenido. "Norma inferior" será, consecuentemente, aquella norma creada de acuerdo con el proceso establecido por la superior $\dot{y}$ con el contenido determinado por esta última.

Conforme a esto, la norma superior determina dos elementos:

a) Determinación procesal: establecimiento del proceso de creación de la norma inferior.

b) Determinación sustancial: establecimiento de los contenidos de las normas inferiores. 
Todas las consideraciones anteriores en este inciso pueden considerarse como la formalización del concepto de la determinación sustancial que de las normas inferiores hace la norma superior.

III.1.2. Consideraciones similares a las anteriores deben hacerse a la postura $r$. Sostiene Anderson que $\mathrm{S}$ puede definirse

$\mathrm{S}={ }_{\mathrm{d} t} \mathrm{KM} \overline{\mathrm{BB}}$

¿Cuál es la significación de esta definición conforme a la semántica que hemos establecido? Hágase la traducción a $A Q J$ y se tiene

$(\mathrm{KMB \overline {B }}) \kappa_{0}$

$\mathrm{K}(\mathrm{MB}) \kappa_{0}(\mathrm{~B}) \kappa_{0}$

$\mathrm{K} \Sigma \kappa_{1} \mathrm{KH} \kappa_{1} \kappa_{0}(\overline{\mathrm{B}}) \kappa_{1}(\mathrm{~B}) \kappa_{0} \quad$ (AQJS)

Anderson [1], p. 178, dice acertadamente que $S$ construida o definida como $\mathrm{KM} \overline{\mathrm{B}} \mathrm{B}$ significa que "la situación indeseable ' $B$ ' se da, y añade la información (sin ningún otra asunción) de que 'B' pudo haber sido evitada".

Nuestra traducción confirma lo dicho por Anderson, pues nuestra fórmula dice que existe un conjunto modelo $\kappa_{1} \mathrm{H}$. alternativo a $\boldsymbol{\kappa}_{0}$ en el que $(\overline{\mathrm{B}})$ existe contenido y que en $\boldsymbol{\kappa}_{0}$ (B) está contenido.

Consecuentemente, $0 p$ se transforma en

$0 \mathrm{p}=\mathrm{LC} \overline{\mathrm{p}} \mathrm{KM} \overline{\mathrm{B}} \mathrm{B}$

$\mathrm{y}$ traduciendo a $\mathrm{AQJ}$

$\mathrm{LC} \overline{\mathrm{p} K M \overline{B B}}=\Pi \kappa_{1} \mathrm{CH} \kappa_{1} \kappa_{0} \mathrm{C}(\overline{\mathrm{p}}) \kappa_{1} \mathrm{~K} \Sigma \kappa_{2} \mathrm{KH} \kappa_{2} \kappa_{1}(\overline{\mathrm{B}}) \kappa_{2}(\mathrm{~B}) \kappa_{1} \quad(\mathrm{AQKS} 0)$

Para todo $\kappa_{1} \mathrm{H}$-alternativo a $\kappa_{0},\left(\mathrm{C}_{\overline{\mathrm{p}}} \mathrm{B}\right) \varepsilon \kappa_{1}$ y existe un $\kappa_{2}$ $H$-alternativo a $\kappa_{1}$ donde $(\bar{B})$ está contenido.

Para Php se tiene

$$
\begin{gathered}
\mathrm{Php}=\mathrm{LCps}=\mathrm{LCpKM \overline {BB }}, \text { pero } \\
\mathrm{LC} \mathrm{pKM} \overline{\mathrm{B} B}=\Pi \kappa_{1} \mathrm{CH} \kappa_{1} \kappa_{0} \mathrm{C}(\mathrm{p}) \kappa_{1} \mathrm{K \Sigma} \kappa_{2} \mathrm{KH} \kappa_{2} \kappa_{1}(\overline{\mathrm{B}}) \kappa_{2}(\mathrm{~B}) \kappa_{1}(\mathrm{AQJSPh})
\end{gathered}
$$


Para todo $\kappa_{1} H$-alternativo a $\kappa_{0}$ si (p) $\varepsilon \kappa_{1}$, entonces existe en $\kappa_{2}$ H-alternativo a $\kappa_{1}$ que contiene (B) y (B) está contenido en $\kappa_{1}$. Por tanto, $(\mathrm{CpB}) \varepsilon \kappa_{1}$.

Para Pp se obtiene, usando transformaciones elementales

$\mathrm{Pp}=\mathrm{MKp} \overline{\mathrm{s}}=\mathrm{MK}_{\mathrm{p}} \overline{\mathrm{K}} \mathrm{M} \overline{\mathrm{B}} \mathrm{B}=\mathrm{MKpALB} \overline{\mathrm{B}} \mathrm{y}$ entonces

$\Sigma \kappa_{1} \mathrm{KH} \kappa_{1} \kappa_{0} \mathrm{~K}(\mathrm{P}) \kappa_{1} \mathrm{~A} \Pi \kappa_{2} \mathrm{CH}_{\kappa_{2}} \kappa_{1}(\mathrm{~B}) \kappa_{2}(\overline{\mathrm{B}}) \kappa_{1} \quad$ (AQJSP)

lo que es fácilmente interpretable.

Aquí surge un problema interesante. Tomemos (AQJSPh). Esta fórmula podría simbolizarse de esta manera:

$$
\begin{array}{r}
\mathrm{Php} \\
\mathrm{CpB} \\
\overline{\mathbf{B}}
\end{array}\left[\begin{array}{l}
\kappa_{0} \\
\kappa_{1} \\
\kappa_{2}
\end{array}\right.
$$

Si en $\kappa_{2}$ tenemos $(\overline{\mathrm{B}})$ por modus tollens, tiene que concluirse que $(\mathrm{CB} \bar{p}) \varepsilon \kappa_{2}$. No es válido concluir que en $\kappa_{2}$ se tenga $(\bar{C} \overline{\mathrm{p}} \overline{\mathrm{B}})$, pues esto no es una conclusión válida.

Lo anterior debe entenderse en el siguiente sentido:

Si el acto antijurídico implica la sanción ( $\mathrm{LCpB})$, es claro que si no se ha aplicado la sanción es porque nó se ha cometido el acto antijurídico. Por tanto, la fórmula

CKKLCpsMs̄sp es válida, lo mismo que

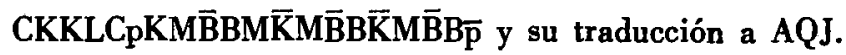

Esto significa que el orden jurídico crea sus propios contenidos; que el establecimiento de los hechos condicionantes de una sanción en una norma jurídica dependiente (por ejemplo la sentencia del juez que sanciona un delito con base en una norma del Código Penal) es una función jurídica creadora. El antecedente de la sanción en la sentencia es establecido por el juez en la sentencia.

Es un error considerar que el delito existe en la 'realidad' y que el juez sólo comprueba su existencia. Tal 'comprobación', en realidad, es constitución, establecimiento o creación del antecedente del condicional definitorio de Php. Tal cons- 
titución del antecedente de la sanción tiene un puesto siste. mático en la semántica desarrollada. Afirmar su existencia en la realidad, es hablar extrasistemáticamente.

Lo anterior puede entenderse en el sentido de que la no aplicación de la sanción $(\overline{\mathrm{B}}) \varepsilon \kappa_{2}$ implica que el acto prohibido no se ha realizado en el ámbito jurídico, es decir, no se ha creado una norma estableciendo la sanción y esto sig. nifica precisamente que no existe jurídicamente acto prohibi. do. El orden jurídico constituye su propio contenido y uno de estos contenidos es el acto prohibido. Éste no está dado y, claro está, no constituye un algo independiente de la norma jurídica. Por el contrario, debe estar puesto por ella.

Este es uno de los argumentos, entre muchos otros, que demuestran el famoso dualismo entre 'ser' y 'deber ser'.

\section{IV}

IV.I. Hasta el momento se ha establecido una lógica deóntica y su semántica que comprende los conceptos de sanción, delito (acto antijurídico o prohibición), obligación y permi. sión. Dicha lógica es el correlato deóntico de la lógica modal $M$ de von Wright o $T$ de Feys. Se ha excluido la posibilidad de la reiteración de operadores deónticos, pues dicha reiteración carece de un sentido claro e intuitivo aceptable. 00p tendría que interpretarse como 'se está obligado a que $P$ sea obligatorio' ó 'es obligatorio que p sea obligatorio'.

Las definiciones de $0, P$ y $\mathrm{Ph}$, tanto en las posturas n.r como en la postura $r$, no permiten interpretar adecuadamente la reiteración de los operadores deónticos mencionados. Debe tenerse especial cuidado en no salirse del contexto establecido en las definiciones de tales operadores, de modo que se les dé una interpretación distinta de aquella establecida en tales definiciones.

Con estos operadores $\mathrm{Ph}, 0$ y $\mathrm{P}$ tan sólo se ha formalizado una parte de los conceptos centrales de la Jurisprudencia. Como la lógica deóntica ha sido establecida por lógicos con hondas preocupaciones éticas, su aplicación al discurso jurí- 
dico ha sido muy escasa. Quizá pueda afirmarse que los operadores mencionados son suficientes para la formalización de ciertos conceptos éticos. Sobre ello no nos pronunciaremos en este trabajo.

Sí diremos, sin embargo, que ellos son totalmente insuficientes para la jurisprudencia, porque en ésta existe y es necesario un concepto nuevo y distinto de los anteriores: el de la facultad.

No siempre se distingue con suficiente claridad entre 'permisión', 'autorización' y 'facultamiento'. Muchas veces se confunden los términos y se usan unos como sinónimos de los otros.

Se puede sin dificultad concebir a la permisión y a la autorización como iguales. Una posible diferencia quizá consista en que la conducta autorizada, cierto, está permitida, pero no lo estaba originalmente. Por el contrario, la conducta ahora autorizada estaba prohibida anteriormente, pero por un acto determinado o por haberse cumplido una condición, la realización de tal conducta no es ya la condición de una sanción. Por ejemplo, conducir un automóvil o ejercer la profesión de médico, en principio, es una conducta prohibida, a menos que exista un acto especial de un órgano del Estado autorizando dicho ejercicio de la profesión o la conducción de un automóvil. Dicho acto especial del órgano del Estado que convierte a esa conducta en permitida consiste en la expedición de una licencia de manejar o en el otorgamiento de un título profesional o patente de médico.

Para evitar imprecisiones en este trabajo no se confundirá entre 'autorización' y 'facultad', aunque somos conscientes que en ocasiones se los utiliza como sinónimos. Se dice a menudo, imprecisamente, para señalar que el órgano legislativo está facultado para crear leyes que está 'autorizado' para ello. Nosotros evitaremos esta terminología.

¿Qué entendemos por facultad? Este concepto señala a aquél contenido de las normas jurídicas que establecen los diversos ámbitos de los actos creadores de normas jurídicas. Es una característica del Derecho, como Kelsen lo ha seña- 
lado, establecer procesos para crear normas jurídicas. El Derecho es un sistema que se autogenera. Los actos creadores de las normas inferiores se encuentran determinados por el contenido de las normas superiores y, estos actos creadores tienen sus ámbitos de validez establecidos:

a) ámbito material: qué acto tiene ese carácter creador,

b) ámbito personal: quién ha de realizar ese acto creador,

c) ámbito temporal: cuando ha de realizarse ese acto creador, $y$

d) ámbito espacial: dónde ha de realizarse ese acto creador.

En otros términos, la facultad es la unidad de aquellos contenidos de normas jurídicas que señalan los ámbitos del proceso creador de otra $u$ otras normas jurídicas.

Un sujeto tiene una facultad ssi (si y sólo si) puede participar en el proceso de creación de una norma, por disposición de la norma superior.

La terminología jurídica se usa de la siguiente manera:

a) al sujeto de la facultad se le denomina 'órgano'

b) a la materia de la facultad se le denomina 'competencia material'

c) al espacio de la facultad se le denomina "jurisdicción espacial'

d) para el tiempo de la facultad se usan conceptos específicos como 'caducidad', 'prescripción', etc.

Tanto $\mathrm{P}$ como $\mathrm{O}, \mathrm{Ph}$ y $\mathrm{S}$ son producto del ejercicio de una F. Una conducta está obligada sólo en el caso de que una norma establezca que su contraria es la condición de una san. ción, y esta norma sólo existe si fue establecida o creada en ejercicio de una $\mathrm{F}$. Y lo mismo con respecto a $\mathrm{Ph}$ y $\mathrm{S}$.

El concepto de facultad señalado no puede reducirse a ninguno de los hasta ahora tratados. En verdad, puede estar unido a cualquiera de ellos, de tal manera que puede acontecer que el ejercicio de una facultad sea obligatorio o constituya una conducta prohibida o, como en general acontece, sea una conducta permitida. 
Si designamos con ' $f$ ' la conducta que constituye el ejercicio de una facultad, los operadores $0, \mathrm{P}, \mathrm{Ph}$ pueden aplicarse a ' $f$ con toda congruencia y claridad.

\section{Véase:}

1) $\operatorname{E} 0 f \overline{\mathrm{P}} \overline{\mathrm{f}} \quad$ - el ejercicio de una facultad es obligatorio ssi su no ejercicio no está permitido

2) EofPh $\bar{f}$ - el ejercicio de una facultad es obligatorio ssi su no ejercicio está prohibido

3) $\mathbf{E P h f \widetilde { P } f}-$ el ejercicio de una facultad está prohibido ssi su ejercicio no está permitido

4) EPhfö - el ejercicio de una facultad está prohibido ssi su no ejercicio es obligatorio

5) EPfō̄ - el ejercicio de una facultad está permitido ssi su no ejercicio no es obligatorio

6) EPfP̄hf - el ejercicio de una facultad está permitido ssi su ejercicio no está prohibido

7) E0fLCfs - el ejercicio de una facultad es obligatorio ssi su no ejercicio implica la sanción

8) EPhfLCfs - el ejercicio de una facultad está prohibido ssi su ejercicio implica la sanción

9) EPfMKfā - el ejercicio de una facultad está permitido ssi es posible ejecutarla sin que se suscite la sanción

10) CofPf - si el ejercicio de una facultad es obligatorio, entonces su ejercicio está permitido

11) CPhf̈Pf - si el no ejercicio de una facultad está prohibido, entonces su ejercicio está permitido

12) CPhfP̄ - si el ejercicio de una facultad está prohibido, entonces su no ejercicio está permitido.

Estos doce teoremas de DM muestran que el concepto de la facultad puede estar unido a cualquiera de los conceptos deónticos $\mathrm{P}, \mathrm{Ph}$ y 0 , con un claro sentido intuitivo, por lo cual podemos suponer que es distinto de ellos.

IV.2. Formalización (DDS4). El esquema para realizar la 
formalización de este concepto de la facultad y los relacionados con él, ya lo tenemos con las posturas n.r y $\mathrm{r}$, expuestas anteriormente; ahora, se dará una interpretación diferente.

Para ello, partimos de una lógica modal deóntica, tal como ha sido definida anteriormente. A esta lógica deóntica DM, se adiciona una constante proposicional ' $\mathrm{N}$ ' cuya negación es posible (que se interpreta como nulidad de una norma, o sea, la negación de una fórmula de $\mathrm{DM}(\overline{\mathrm{Pa}}, \overline{\mathrm{O}} \mathrm{a}, \overline{\mathrm{P}} \mathrm{ha})$; a las fórmulas $\mathrm{DM}$ en conjunto simbolizaremos por $\mathrm{T} \alpha$ ) y se define F como:

$$
\mathrm{FT} \alpha={ }_{\mathrm{df}} \mathrm{MKT} \alpha \overline{\mathrm{N}}
$$

De aquí se puede definir:

$$
\begin{array}{lll}
\overline{\mathrm{F} T} \alpha={ }_{\mathrm{df}} \mathrm{LCT} \alpha \mathrm{N} & \text { y } \\
\overline{\mathrm{F}} \overline{\mathrm{T}} \alpha={ }_{\mathrm{df}} \mathrm{LCT} \alpha \mathrm{N} & & \text { (C.I) } \\
&
\end{array}
$$

¿Cuáles son las consideraciones intuitivas subyacentes a esta formalización?

Paralelamente a lo que acontece en DM, en esta formalización, a la que denominaremos DDS4, para conseguir que ella sea una extensión de una lógica modal alética, tenemos que introducir una constante proposicional ' $N$ ' cuya negación es posible: $M \bar{N}$. ' $N$ ' se interpreta como la nulificación de un acto creador de una norma, o lo que es igual, la nulificación de una norma, la negación de su validez o el establecimiento de su invalidez. Y, añadiremos, la información de que esta nulidad puede no llevarse a cabo.

Como 'T $\alpha$ ' está por $0 p$, Php ó Pp, (C.F) dice:

$F T p=M K T p N-$ la facultad para hacer $p, T(0, P h, P)$ significa que es posible dictar la norma TP y ésta no se nulifique.

La condición (C.I) señala el caso de la incompetencia o no facultamiento: 
$F T p=L C T p N-$ no tener la facultad para hacer $p, T(0, P h, P)$ significa que es necesario que si se dicta $T p$, entonces se nulifique Tp.

La condición (C.Q) señala el caso de la incompetencia o no facultad para $\overline{\mathrm{T}} \mathrm{a}$ :

$\overline{\mathrm{FT}} \mathrm{p}=\mathrm{LC} \overline{\mathrm{T}} \mathrm{pN}-$ no tener la facultad para hacer $\mathrm{p}, \overline{\mathrm{T}}(\overline{0}, \overline{\mathrm{P}} \mathrm{T}, \overline{\mathrm{P}})$ significa que es necesario que si se dicta $\mathrm{T} p$, entoncès se nulifique $T$ p.

Siguiendo el esquema de A. R. Anderson y con el fin de crear una lógica deóntica de los operadores dinámicos que sea normal, definimos a $\mathrm{N}$ de la siguiente manera:

(N) $\quad \mathrm{N}={ }_{d f} K M T p T p$

La definición $(N)$ nos indica que la regularidad de Tp es verdadera pero que es posible que Tp se nulifique: MT $\mathbf{T}$. La nulificación de Tp puede ser evitada evidentemente, si se dicta una norma regular Tp. La estructura formal es exactamente paralela a la de la sanción en el sistema de Anderson. Aunque aquí se dé una interpretación díferente, que mostraremos, primeramente, con la interpretación de $\mathrm{IT} p=\overline{\mathrm{FT}} \mathrm{p}$

(1) $\operatorname{IT}_{\mathrm{p}}=\overline{\mathrm{F}} \mathrm{T} p=L C T \mathrm{pN}$

(1) indica, como ya fúe señalado, que ser incompetente, i.e., no estar facultado a dictar la norma $T p$, significa que es necesario que si se dicta la norma $T p$, entonces, ésta se nulifique. $\mathrm{N}$ es la consecuencia jurídica de la irregularidad de la norma.

Si se hace la sustitución de $(N)$ en $(1)$, se obtiene:

(2) $I T p=L C T p K M T \bar{T} T p$

(2). indica que es necesario que si se dicta $\mathrm{Tp}$, entonces la nulificación de Tp es posible y $\mathrm{T} p$, como norma regular, es verdadera. 
En el caso de:

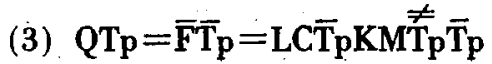

se afirma que no estar facultado para dictar $\overline{\mathrm{T}}$, o sea, ser incompetente o no tener la facultad para dictar $\mathrm{T} p$, significa que es necesario que si se dicta $\bar{T}$ p, entonces es posible que se dicte la norma $\mathrm{T} p$, nulificadora de $\overline{\mathrm{T}} \mathrm{p}$ y señala que $\overline{\mathrm{T}} \mathrm{p}$ se ha dictado, lo que significa que es regular (aunque provisionalmente).

Las consideraciones intuitivas que subyacen en todo esto son las siguientes:

En primer lugar, y esto debe ser enfatizado, el orden jurídico es un orden dinámico positivo, en el sentido afirmado por la teoría de Kelsen: 'Ningún imperativo sin Imperator'. Toda norma jurídica es una norma positiva. Se rechaza, por tanto, el iusnaturalismo, en tanto doctrina que afirma la existencia de normas que no han sido establecidas por actos humanos, porque existen inmanentes en la naturaleza, en general, en el hombre o su razón. Por lo tanto, esto implica el rechazo de la razón práctica, de la razón legisladora.

En segundo lugar, y como consecuencia de lo anterior, las distintas ocurrencias de $\mathrm{Tp}$ o $\overline{\mathrm{T}} \mathrm{p}$ deben considerarse como enunciados de la ciencia jurídica que ha comprobado que normas tales se han dictado por las autoridades jurídicas. Esto no excluye, evidentemente, prognosis sobre lo que juridicamente es posible, con tal de que se distinga pulcramente entre los enunciados de la jurisprudencia o metajurisprudencia, y las normas emitidas o creadas por los órganos jurídicos. Y éste es, precisamente, el sentido que puede dársele al concepto de la interpretación jurisprudencial de las normas jurídicas.

Debe resultar evidente para cualquier jurista que la ciencia del Derecho no puede crear normas jurídicas, sino su función consiste en la determinación sistemática, con arregic a principios, de los contenidos de las normas jurídicas. Este ordenamiento sistemático del contenido de las normas jurídicas positivas se realiza, principalmente, en la determinación 
del sentido de los contenidos normativos. Por esto entiendo la explicitación de las expresiones lingüísticas de las normas y su interrelación congruente (no contradictoria), lo que comprende la determinación posible (y recalco, posible) de los contenidos que pueden tener las normas inferiores que habrán de crear los órganos jurídicos con base en esas normas mencionadas.

Esto es exactamente lo que hacen nuestros operadores F,I y Q. Señalan las condiciones a las que se encuentra sujeta la jurisprudencia al realizar su prognosis interpretativa. Dada una interpretación, el desarrollo de la fórmula con nuestro cálculo indica o señala las diversas posibilidades de prognosis.

Analicemos (2) en la forma siguiente:

(2') IOp $=$ LCOpKM $\overline{0} \mathrm{p} 0 \mathrm{p}$

Op es una fórmula deóntica perteneciente a DM. Para nuestros efectos no es necesario desarrollarla en (AQJS0). Por lo pronto hay que observar que $\left(2^{\prime}\right)$ es un enunciado formalizado de la jurisprudencia positiva. Dice que es necesario que si se ha dictado una norma que establece que es obligatorio que $p$, entonces es posible que la norma que establece como obligatorio que $p$ se nulifique por irregular e indica, además, que es regular, por lo pronto, que sea obligatorio que p. Su traducción metalingüística (AQJNI) nos despliega lo anterior:

$$
\Pi \kappa_{1} \mathrm{CH} \kappa_{1} \kappa_{0} \mathrm{C}(0 \mathrm{p}) \kappa_{1} \mathrm{~K} \sum \kappa_{2} \mathrm{KH} \kappa_{2} \kappa_{1}(\overline{0 p}) \kappa_{2}(0 \mathrm{p}) \kappa_{1}
$$

Obsérvese que hay dos ocurrencias de $(0 p) \kappa_{1}$, mientras que $(\overline{0} p) \kappa_{2}$ sólo tiene una ocurrencia. $(0 p) \kappa_{1}$, en su primera ocurrencia nos señala que se ha dictado la norma que hace a p obligatorio. Conjuntivamente con esta determinación se señala:

1) Existe un $\kappa_{2}$ en el que 0p está contenido, es decir, puede existir una norma que nulifique a $0 p$ y 
2) La segunda ocurrencia de $(0 p) \kappa_{1}$, señala que $0 p$ es una norma válida, regular.

(AQJNI) no indica bajo qué condición 0p puede ser nulificada, pero sí indica que esta nulificación es posible. Pero, por el uso del modus tollens, esta fórmula señala que en caso de que (0p) $\kappa_{1}$ se nulifique, o sea, de que se dicte una norma $(\overline{0} p) \kappa_{2}$, esto significa que $(0 p) \kappa_{1}$ fue irregular, pues es válida la fórmula

\section{(4) CKKLCOpNMN̄NO$\overline{0}$}

$\mathrm{y}$, por tanto, es válida la fórmula

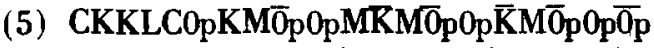

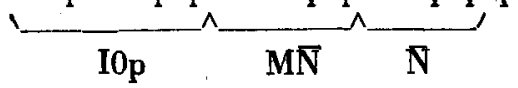

Exactamente puede decirse lo mismo por lo que respecta a Q0p, puesto que

$$
\begin{aligned}
Q 0 p & =F \bar{p} \\
\text { Pero } \mathrm{FT} \alpha & =\mathrm{IT} \alpha, \text { por lo que } Q 0 \mathrm{p}=\mathrm{I} \overline{\mathrm{O}} \mathrm{p}
\end{aligned}
$$

y todo lo que tiene que hacerse es substituir en (4) y (5) $0 p$ por $\overline{0}$.

La única dificultad de interpretación se presenta, en realidad, con FTp. La dificultad que señalaremos surge de que en FTp aparece $\bar{N}$, o lo que es lo mismo, $\overline{\mathrm{K}} \mathrm{MT}$ pTp.

$\mathrm{Si}$ se hacen transformaciones elementales se obtiene:

$$
\overline{\mathbf{K}} \mathbf{M} \overline{\mathrm{T}} \mathbf{p} \mathrm{T} p=\operatorname{ALT} \stackrel{\neq}{\mathbf{T}} \bar{p}
$$

lo que expresa que es necesario $\mathrm{Tp}$ o que $\mathrm{Tp}$ es nulo, i.e., no es verdadero.

Pero esto no debe sorprender o extrañar, pues KMTpTp señala que la nulidad, como consecuencia de la irregularidad, es posible. Entonces, $\overline{\mathrm{K}} \mathrm{M} \overline{\mathrm{T}} \mathrm{p} \mathrm{T}$, es decir, la negación de $\mathrm{N}$, tiene que señalar, como un rodeo poco intuitivo, que ó $\mathrm{T}_{\mathrm{p}}$ es necesario ó $\overline{\mathrm{T}} \mathrm{p}$ es verdadero, es decir, es nulo, lo que sabe- 
mos de antemano que es falso. Por lo que FTp tan sólo nos importa el Tp que aparece en un principio como norma dictada en el ejercicio de la facultad. Lo que, además, puede indicar que en el ejercicio de una facultad puede dictarse Tp o no puede dictarse $T p$, pues la facultad simplemente autoriza a dictar una norma, sin que exista la compulsión de cualquier tipo, de dictarla.

(6) $\mathrm{FTp}=\mathrm{MKTpN}$

Es posible dictar la norma Tp y que ésta no se nulifique, resulta evidente. Pero

(7) $\operatorname{MKTp} \overline{\mathrm{N}}=\mathrm{MKTpK} M \overline{\mathrm{T}} \mathrm{pTp}=\mathrm{MKTpALTp} \overline{\mathrm{T}}_{\mathrm{p}}$

'Si se hace. su transformación metalingüística (AQJNF) se obtiene:

(8) $\Sigma \kappa_{1} \mathrm{KH} \kappa_{1} \kappa_{0} \mathrm{~K}(\mathrm{Tp}) \kappa_{1} \mathrm{AII} \kappa_{2} \mathrm{CH} \kappa_{2} \kappa_{1}(\mathrm{Tp}) \kappa_{2}(\mathrm{Tp}) \kappa_{1}$

Si se obtiene la estructura proposicional de (7) y (8), es decir, sin la presencia de los operadores modales, se ve que tiene esta estructura:

\section{KaAaã}

si 'a' se sustituye por $\mathrm{Tp}$, y es claro que de ambas premisas a y $A a \bar{a}$, se puede concluir siempre válidamente que a. Con operadores modales es válida en DM la siguiente fórmula:

(9) CMKTpALTpTpMTp

Con nuestra semántica, se concluye que es válido dictar la norma $\mathrm{Tp}$, que era lo interesante.

La traducción de (9) a (AQJNF) lo demuestra:

$\mathrm{C} \Sigma \kappa_{1} \mathrm{KH} \kappa_{1} \kappa_{0} \mathrm{~K}(\mathrm{Tp}) \kappa_{1} \mathrm{AII} \kappa_{2} \mathrm{CH} \kappa_{2} \kappa_{1}(\mathrm{Tp}) \kappa_{2}(\overline{\mathrm{T}} \mathrm{p}) \kappa_{1} \Sigma \kappa_{1} \mathrm{KH} \kappa_{1} \kappa_{0}(\mathrm{Tp}) \kappa_{1}$

pues ésta es una fórmula cuantificacional válida si tiene como antecedente $\Pi \lambda H \lambda \lambda$.

Con todo lo anterior, pueden hacerse consideraciones aná- 
logas a las hechas anteriormente, por lo que respecta al acto prohibido en su relación con la sanción y en aplicación del modus tollens, pero en este caso con aplicación a la irregularidad en la creación de una norma. Así como no hay delito sino hay la sanción, así no puede predicarse la irregularidad de una norma si no es con base en otra que la nulifique. No hay normas nulas a priori, sino tan sólo normas anulables o anuladas, es decir, cuando ya no hay norma.

El Derecho crea su propio contenido y expulsa a los elementos disolventes o irregulares del mismo.

Es un paso exigido preguntarse sobre la reiteración de estos operadores F, I y Q.

¿Tiene algún sentido la fórmula FFTp? La contestación es positiva, pues FFTp expresa el caso de una delegación pura de facultades, en el que el órgano 1 delega su facultad a dictar Tp a un órgano 2. Lo mismo valdría para el caso FFFTp y demás combinaciones posibles. Esto constituye el fundamento del concepto de la jerarquía del orden jurídico, con la ventaja de que desarrollando las fórmulas, muy com. plicadas del (AQJN), se obtienen todas las posibilidades de irregularidad y nulificación en los diversos grados del orden jurídico.

En DDS4 valen las siguientes definiciones:

I) $\mathrm{QT} \alpha=\mathrm{FT} \alpha$

II) $\mathbf{F T} \alpha=\overline{\mathrm{QT}} \alpha$

III) $\overline{\mathrm{T}} \alpha=\mathrm{F} \mathbf{T} \alpha$

IV) $\bar{F} \mathrm{~T} \alpha=\mathrm{QT} \alpha$

Entonces, pueden organizarse de tal manera las cosas, que se obtengan todas las combinaciones posibles entre los ope. radores dinámicos de las definiciones (I) -(IV) y los operadores deónticos 0 y $P$ en unión con la descripción de la conducta respectiva o su omisión p ó $\overrightarrow{\mathrm{p}}$. Por ello formamos una matriz cuyas líneas sean $Q, \bar{F}, \bar{Q}, \bar{F}$ y cuyas columnas estén formadas por $0 p, P p, 0 \bar{p}, P \bar{p}, \overline{0}, \bar{P}_{p}, \overline{0} \bar{p}, \bar{P}_{\bar{p}}$ (véase Tabla II). 
Tabla II

\begin{tabular}{|c|c|c|c|c|c|c|c|c|}
\hline & $0 p$ & $\mathbf{P p}_{\mathbf{p}}$ & $0 \bar{p}$ & $\mathbf{P}_{\overline{\mathbf{p}}}$ & $\overline{0}_{p}$ & $\overline{\mathbf{P}}_{\mathbf{p}}$ & $\overline{0} \bar{p}$ & $\overline{\mathbf{P}}_{\overline{\mathbf{p}}}$ \\
\hline $\mathbf{Q}$ & $\begin{array}{l}\text { QOp } \\
\text { (1) }\end{array}$ & $\underset{\text { (2) }}{\mathrm{QPp}}$ & $\underset{(3)}{Q 0 \bar{p}}$ & $\underset{(4)}{Q P \bar{p}}$ & $\begin{array}{l}Q \overline{0} p \\
(5)\end{array}$ & $\underset{(6)}{Q \bar{P} p}$ & $\begin{array}{l}\mathrm{Q}_{\overline{0}}^{\bar{p}} \\
(7)\end{array}$ & $\underset{(8)}{Q_{\bar{P}} \bar{p}}$ \\
\hline $\mathbf{F}$ & $\underset{(9)}{F 0 p}$ & (10) & $\begin{array}{l}\text { Fo } \bar{p} \\
\text { (11) }\end{array}$ & $\underset{(12)}{F P \bar{p}}$ & $\begin{array}{l}\text { Fop } \\
(13)\end{array}$ & $\underset{(14)}{F \bar{P} p}$ & $\begin{array}{l}\bar{F} \overline{0} \bar{p} \\
(15)\end{array}$ & $\begin{array}{l}F \bar{P} \bar{p} \\
(16)\end{array}$ \\
\hline$\overline{\mathbf{Q}}$ & $\begin{array}{l}\bar{Q} 0 p \\
(17)\end{array}$ & $\begin{array}{l}\overline{\mathrm{QPp}} \\
\text { (18) }\end{array}$ & $\begin{array}{l}\bar{Q} 0 \bar{p} \\
(19)\end{array}$ & $\begin{array}{l}\overrightarrow{\mathrm{QP}} \overline{\mathrm{p}} \\
(20)\end{array}$ & $\begin{array}{l}\overline{\mathrm{Q}} \overline{0_{p}} \\
(21)\end{array}$ & $\begin{array}{l}\overline{Q P}_{(22)} \\
(2)\end{array}$ & $\begin{array}{l}\overline{\mathrm{Q}} \overline{\overline{\mathrm{p}}} \\
(23)\end{array}$ & $\begin{array}{l}\overline{\mathrm{QP}} \overline{\mathrm{p}} \\
(24)\end{array}$ \\
\hline$\overline{\boldsymbol{F}}$ & $\begin{array}{l}\overline{\mathrm{F}} 0 \mathrm{p} \\
(25)\end{array}$ & $\underset{(26)}{\bar{F} P p}$ & $\begin{array}{l}\overline{\mathrm{F}} 0 \bar{p} \\
\text { (27) }\end{array}$ & $\begin{array}{c}\overline{\text { FPp }} \\
\text { (28) }\end{array}$ & $\begin{array}{l}\overrightarrow{F O p} \\
(29)\end{array}$ & $\underset{(30)}{\overline{\mathbf{F}} \overrightarrow{\mathbf{P}}_{\mathbf{p}}}$ & $\begin{array}{l}\overline{\mathrm{F}} \overline{0} \overline{\bar{p}} \\
\text { (31) }\end{array}$ & $\frac{\bar{F}_{\overline{\mathbf{P}}}}{(32)}$ \\
\hline
\end{tabular}

Haciendo las transformaciones elementales, nos encontramos con que existen 8 independientes, pues las demás pueden reducirse a esas ocho:

Tabla III

\begin{tabular}{|lll|}
\hline & (a) $\quad$ (b) $\quad$ (c) $\quad$ (d) \\
1. $Q 0 \mathrm{p}=(1)=(8)=(28)=(29)$ \\
2. $Q \mathrm{QP}=(2)=(7)=(27)=(30)$ \\
3. $\mathrm{QQp}=(3)=(6)=(26)=(31)$ \\
4. $\mathrm{QP}=(4)=(5)=(25)=(32)$ \\
5. $\mathrm{FOp}=(9)=(16)=(20)=(21)$ \\
6. $\mathrm{FPp}=(10)=(15)=(19)=(22)$ \\
7. & $\mathrm{F} 0 \overline{\mathrm{p}}=(11)=(14)=(18)=(23)$ \\
8. & $\mathrm{FP} \overline{\mathrm{p}}=(12)=(13)=(17)=(24)$ \\
\hline
\end{tabular}

Si se sigue el orden señalado en cada una de las líneas 1 a 8 y se obtienen las transformaciones en lógica secuencia. Es decir, 1 se transforma en 8, ésta en (28) y ésta en (29).

Tablas similares podrían hacerse para el caso de que existieran reiteraciones de los operadores dinámicos. 
El cálculo establecido con su interpretación semántica ba. sada en los conjuntos modelo de Hintikka y su traducción metalingüística, corresponde al sistema lógico modal S4, pues la relación de $\mathrm{H}$-alternatividad es, en este caso, transitiva, porque la fórmula característica de $\mathrm{S} 4, \mathrm{CMMpMp}$, es válida en el cálculo establecido, en la forma siguiente:

\section{CFFTpFTp}

Por ello, este cálculo lo hemos denominado DDS4.

Como observación final deseo expresar que en el libro de Snyder [7], se encontrará material y técnica para desarrollar la teoría aquí presentada, incluso dentro de AQN. Queda por presentar el sistema de cancelación correspondiente. Además, puede intentarse la integración de las (AQJS) y (AQJN), es decir, de DM y DDS4, así como la interpretación intuitiva detallada y minuciosa, con sus aplicaciones a la Jurisprudencia positiva en campos concretos.

\section{REFERENCIAS}

[1] Anderson, A. R. "The Formal Analysis of Normative Systems" en Rescher, N. (ed.), The Logic of Decision and Action. Pittsburgh: University of Pittsburgh Press, 1967, pp. 147-213.

[2] Follesdal, Dagfinn y Hilpinen, Risto. "Deontic Logic: An Introduction" en Hilpinen, R. (ed.), Deontic Logic: Introductory and Systematic Readings. Dordrecht-Holland: D. Reidel Publishing Company, 1971.

[3] Hintikka, Jaakko. "Some Main Problems of Deontic Logic" en Hilpinen, R. (ed.), Deontic Logic: Introductory and Systematic Readings. DordrechtHolland: D. Reidel Publishing Company, 1971.

[4] Hughes, G. E. y Cresswell, M. J. An Introduction to Modal Logic. London: Methuen and Co., Ltd., 1968.

[5] Kelsen, Hans. Reine Rechtslehre. 2a. edición. Viena: Franz Deutike, 1960.

[6] Teoría general del Estado. Traducción de Luis Legaz y Lacambra. México: Editora Nacional, S. A., 1948.

[7] Snyder, D. Paul. Modal Logic and its Applications. New York: Van Notrand Reinhold Company, 1971. 
SUMMARY

In this paper, Schmill begins by systematizing the different approaches that have been offered in order to formalize a certain set of concepts, namely, the normative concepts. Two main approaches are outlined by Schmill: the 'reductionist' and the 'non-reductionist' approaches. The 'reductionists' claim that deontic logic can be reduced to alethic modal logic (A. R. Anderson). Those adhering to the 'non-reductionist' approach claim that deontic logic is construed either by introducing the $\mathrm{O}$ - and $\mathrm{P}$ - operators ex novo into propositional logic or by adding a propositional constant ' $S$ ' (which may be interpreted as a sanction) and an axiom stating the possibility of the negation of ' $S$ ' $(\mathbf{M} \bar{s})$ to alethic modal logic.

Schmill goes on to describe the semantics of the formal systems belonging to both the reductionist and non-reductionist approaches using Hintikka's notions of model sets and model systems. He then offers an interpretation of the sentences belonging to the object language (the formulas of modal logic) as well as of those sentences belonging to the metalanguage (sentences which belong to the semantics of the formal system under consideration). The former sentences are considered part of Positive Jurisprudence, while the latter are supposed to constitute what has traditionally been called General, or Pure, Jurisprudence.

As has been seen, the interpretation of sentences of modal logic is carried out by means of model sets. In his interpretation, however, Schmill considers the latter to be descriptions of norms belonging to some particular, undetermined legal system. As for the interpretation of the sentences of the metalanguage, that is, those sentences which state the membership relation between formulas of modal logic and model sets, Schmill considers the relation that holds between model sets as a dependence relation between the legal propositions which they represent. Hence, those conditions which determine the criteria of material consistency of the deontic content of the legal propositions dependent on a specific legal proposition are specified.

The analysis of the concepts of obligation, permission and prohibition has proven to be insufficient in Jurisprudence. Therefore, we have to enrich our analysis by introducing a new concept, that of a faculty (legal power). This concept has often been confused with that of permission or that of authorization. In order to avoid 
misunderstandings, Schmill defines the concept in the following way: a faculty refers to that content of legal norms which states the different spheres of the norm-creating acts. Therefore we are allowed to say that an individual has a faculty if and only if he is allowed to participate in the norm-creating process by disposition of a higher (legal) norm.

Since, following Hans Kelsen, we can say that a behavior is obligatory only if a legal norm attaches a coercive act, or sanction, to the opposite behavior and that this norm exists only because it has been created by the exercise of a faculty, it seems clear that oblig. ations, as well as permissions or prohibitions, may be considered products of the exercise of a faculty. Furthermore, we could also say that the exercise of a faculty may fall under the scope of any other deontic operator, that is, it could be obligatory, permitted or forbidden.

Once the concepts of faculty, competence and nullity have been formalized, Schmill shows how the forecasting function of Jurisprudence is subject to the conditions which these operators determine. Finally, Schmill gives an affirmative answer to the question of the possibility of iterating these operators, treating this case as a power-conferring one.

(Summary by Alvaro Rodríguez Tirado) 